\title{
A New Five - Parameter Lifetime Model: Theory and Applications
}

\author{
Abdullah Aljouiee \\ Department of Mathematics and Statistics \\ Al Imam Mohammad Ibn Saud Islamic University, Saudi Arabia \\ amjouiee@imamu.edu.sa \\ Ibrahim Elbatal \\ Department of Mathematics and Statistics \\ Al Imam Mohammad Ibn Saud Islamic University, Saudi Arabia (IMSIU) \\ iielbatal@imamu.edu.sa \\ Hazem Al-Mofleh \\ Department of Mathematics \\ Tafila Technical University, Tafila, 66110 Jordan \\ almof1hm@cmich.edu
}

\begin{abstract}
In this paper we defined a new lifetime model called the the Exponentiated additive Weibull (EAW) distribution. The proposed distribution has a number of well-known lifetime distributions as special submodels, such as the additive Weibull, exponentiated modified Weibull, exponentiated Weibull and generalized linear failure rate distributions among others. We obtain quantile, moments, moment generating functions, incomplete moment, residual life and reversed Failure Rate Functions, mean deviations, Bonferroni and Lorenz curves. The method of maximum likelihood is used for estimating the model parameters. Applications illustrate the potentiality of the proposed distribution.
\end{abstract}

Keywords: Exponentiated additive Weibull, Moments, Modified Weibull Distribution, Maximum likelihood estimation.

\section{Introduction}

In reliability engineering and lifetime analysis many applications require a bathtub shaped hazard rate function. Weibull distribution is one of the most commonly used lifetime distributions in reliability and lifetime data analysis. It is flexible in modeling failure time data, as the corresponding hazard rate function can be increasing,constant or decreasing. But in many applications in reliability, mechanical and electronic components and survival analysis, the hazard rate function can be of bathtub shape. It is well known that, because of design and manufacturing problems, the hazard rate is high at the beginning of a product life cycle and decreases toward a constant level. After reaching a certain age, the product enters wear-out phase and hazard rate starts to increase. Despite the fact that this phenomenon has been presented in many reliability engineering texts few practical models possessing this property have appeared in the literatures twoparameter flexible Weibull extension of Bebbington et al. (2007) has a hazard function that can be increasing, decreasing or bathtub shaped. Zhang and Xie (2011) studied the characteristics and application of the truncated Weibull distribution which has a bathtub shaped hazard function. 
A three parameter model, called exponentiated Weibull distribution, was introduced by Mudholkar and Srivastave (1993). Xie and Lai (1995) introduced a four-parameter distribution called the additive Weibull distribution based on the simple idea of combining the hazard rates of two Weibull distributions: one has a decreasing hazard rate and the other one has an increasing hazard rate. It has the cumulative distribution function is given by

$$
F(x, \alpha, \theta, \mu, \beta)=1-e^{-\left(\alpha x^{\theta}+\mu x^{\beta}\right)} ; \quad x>0,
$$

where $\alpha>0, \mu>0$ and $\theta>\beta>0$, or $\beta>\theta>0$ which gives identifiability to the model, when $\theta>0$ the hazard rate is increasing and when $0<\beta<1$ hazard rate is decreasing. The corresponding probability density function is

$$
f(x, \alpha, \theta, \mu, \beta)=\left(\alpha \theta x^{\theta-1}+\mu \beta x^{\beta-1}\right) e^{-\left(\alpha x^{\theta}+\mu x^{\beta}\right)},
$$

where $\alpha>0$ and $\mu>0$ are scale parameters, and $\theta>\beta>0$, or $(\beta>\theta>0)$ are shape parameters. The interpretation of model (2) is evident. Suppose a system composed of two interconnected independent series sub-systems that affect the system in a different way, each one having a Weibull distribution with proper parameters. The hazard time of the system follows (2), since it occurs when the first of the two sub-systems fails.

Since 1995, exponentiated distributions have been widely studied in statistics and numerous authors have developed various classes of these distributions. A good review of some of these models is presented by Pham and Lai (2007). The exponentiation of distributions is a mechanism that makes the model more flexible, Nadarajah and Kotz (2006) introduce four more exponentiated type distributions: the Exponentiated Gamma, Exponentiated Weibull, exponentiated Gumbel and the Exponentiated Fréchet distribution. There are also several authors presented exponentiated distributions, such as Barriga, Louzada and Cancho (2011) with the Complementary Exponential Power distribution which is the exponentiation of the Exponential Power distribution proposed by Smith and Bain (1975) denoted as Complementary Exponential Power distribution, Bakouch, Al-Zahrani, Al-Shomrani, Marchi and Louzada (2011) with the extension of the Lindley (EL) distribution and the Complementary Exponential Power distribution (CEP) introduced by Barriga, Louzada and Cancho (2011).

In this paper, the so-called exponentiated additive Weibull (EAW) distribution with five parameters is proposed. The new distribution due to its flexibility in accommodating all the forms of the hazard rate function can be used in a variety of problems for modeling lifetime data. Another important characteristic of the distribution is that it contains, as special sub-models, the Weibull, exponentiated exponential (Gupta and Kundu, 1999, 2001), exponentiated modified Weibull distribution (Elbatal, 2009), exponentiated Weibull distribution (Mudholkar et al., 1995, 1996), generalized linear failure rate distribution, (Sarhan and Kundu, 2009), Modified Weibull distribution (Lai et al, 2003), among some other distributions. The exponentiated additive Weibull distribution is not only convenient for modeling comfortable bathtub-shaped failure rates data but is also suitable for testing goodness-of-fit of some special sub-models such as the exponentiated Weibull and modified Weibull distributions. 
The rest of the article can be organized as follows. In section 2 we present the expression of the pdf and cdf of the subject distribution and some special sub-models. In section 3 we study the statistical properties including moments, moment generating function and incomplete moments. Residual life and reversed residual functions of the $E A W$ distribution, Bonferroni and Lorenz curves and mean deviations are discussed in Section 4. In section 5 we demonstrate the maximum likelihood estimates of the unknown parameters. Simulation results to assess the performance of the maximum likelihood estimation method are reported in Section 6. Finally, in section 7 we present a data analysis to illustrate the usefulness of the proposed distribution.

\section{Exponentiated Additive Weibull Distribution}

A random variable $X$ has the $E A W$ distribution with parameter vector $\phi=(\alpha, \theta, \mu, \beta$ $, \lambda)^{T}$ say, $E A W(\boldsymbol{\phi})$, or $E A W(\alpha, \theta, \mu, \beta, \lambda)$ if its cumulative distribution given by

$$
F(x, \boldsymbol{\phi})=\left[1-e^{-\left(\alpha x^{\theta}+\mu x^{\beta}\right)}\right]^{\lambda} ; \quad x>0,
$$

and its probability density function given by

$$
f(x, \boldsymbol{\phi})=\lambda\left(\alpha \theta x^{\theta-1}+\mu \beta x^{\beta-1}\right) e^{-\left(\alpha x^{\theta}+\mu x^{\beta}\right)}\left[1-e^{-\left(\alpha x^{\theta}+\mu x^{\beta}\right)}\right]^{\lambda-1}
$$

The survival function, also known as the reliability function (rf) in engineering, is the characteristic of an explanatory variable that maps a set of events, usually associated with mortality or failure of some system onto time. The corresponding survival function of random variable $X$ is

$$
\bar{F}(x, \boldsymbol{\phi})=1-\left[1-e^{-\left(\alpha x^{\theta}+\mu x^{\beta}\right)}\right]^{\lambda},
$$

and the hazard rate (failure) function (hrf) which is an important quantity characterizing life phenomenon functions takes the following form

$$
h(t)=\frac{f(t, \boldsymbol{\phi})}{\bar{F}(t, \boldsymbol{\phi})}=\frac{\lambda\left(\alpha \theta t^{\theta-1}+\mu \beta t^{\beta-1}\right) e^{-\left(\alpha t^{\theta}+\mu t^{\beta}\right)}\left[1-e^{-\left(\alpha t^{\theta}+\mu t^{\beta}\right)}\right]^{\lambda-1}}{1-\left[1-e^{-\left(\alpha t^{\theta}+\mu t^{\beta}\right)}\right]^{\lambda}} ; t>0
$$

whereas its reversed hazard rate (failure) function (rhf) is given by

$$
\tau(t)=\frac{f(t, \boldsymbol{\phi})}{F(t, \boldsymbol{\phi})}=\frac{\lambda\left(\alpha \theta t^{\theta-1}+\mu \beta t^{\beta-1}\right) e^{-\left(\alpha t^{\theta}+\mu t^{\beta}\right)}}{\left[1-e^{-\left(\alpha t^{\theta}+\mu t^{\beta}\right)}\right]} ; t>0 .
$$

Figure 1 provides some plots of the density curves for different values of the parameters $\alpha, \theta, \mu, \beta$ and $\lambda$.

Figure 2 provides the same for some plots of the hrf, it is showing that the $E A W$ distribution is quite flexible for modelling survival data. 


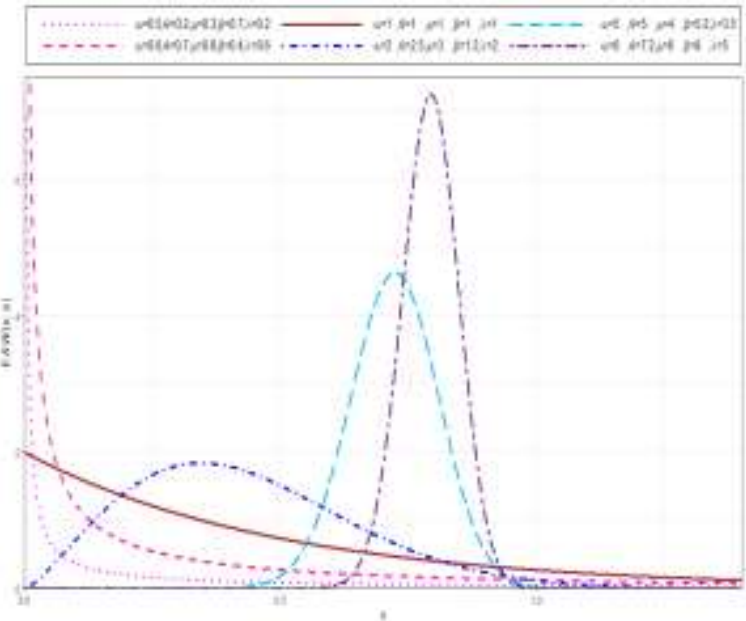

(a)

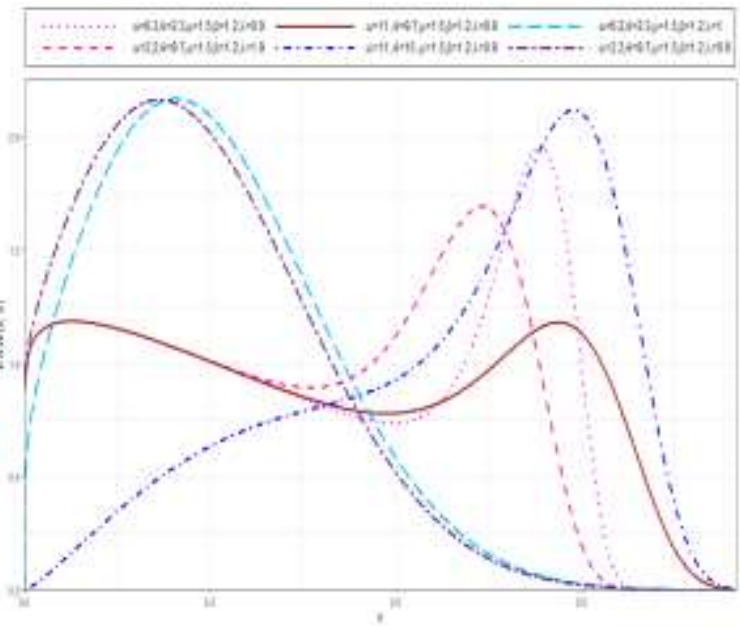

(b)

Figure 1: Plots of the $E A W$ distribution for some parameter values.

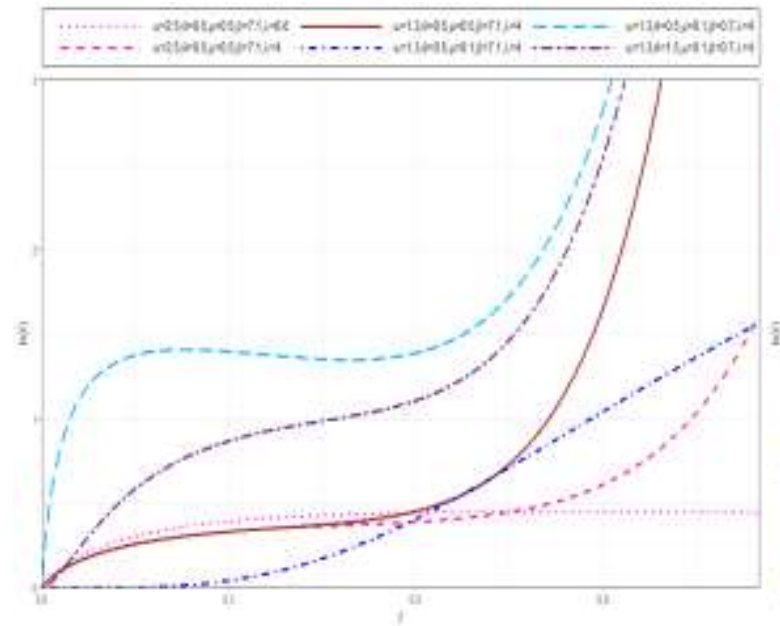

(a)

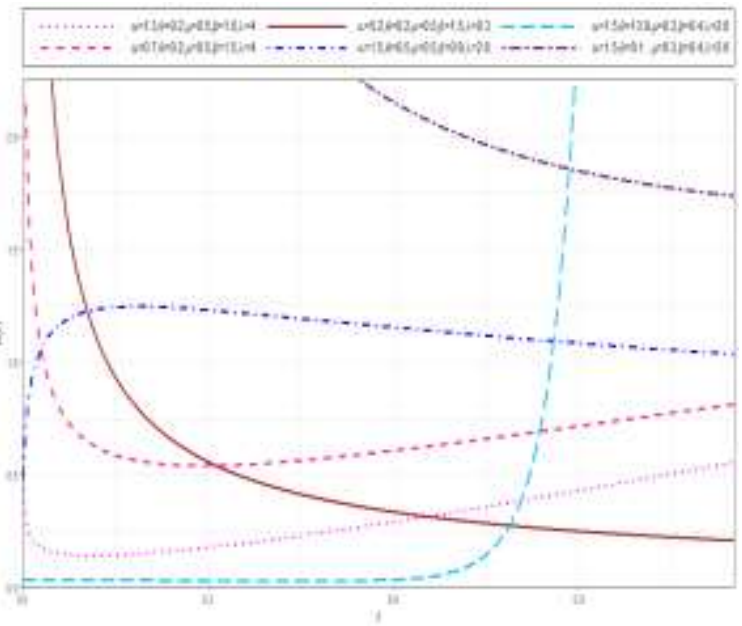

(b)

Figure 2: The $E A W$ hazard rate function for some parameter values.

It is known, not many lifetime distributions exhibit bathtub hazard rates. The $E A W$ model shows flexibility in accommodating all forms of the hazard rate function as seen from Figure 2 (by changing its parameter values) seems to be an important distribution that can be used.

\subsection{Special Cases of the $E A W$ Distribution}

The exponentiated additive Weibull distribution is a very flexible model that approaches to different distributions when its parameters vary. The flexibility of the exponentiated additive Weibull distribution is explained in Table 1. Note that in the table we have used the following abbreviations: 
$A=$ Additive $; G=$ Generalized $; \quad M=$ Modified,$\quad W=$ Weibull, $\quad E_{x}=$ Exponential, $E=$ Exponentiated, $L F R=$ Linear Failure Rate and $R=$ Rayleigh. If $X$ is a random variable with cdf (3), then we have the following cases:

Table 1: The sub-models of the $E A W$ distribution

\begin{tabular}{||l||c|c|c|c|c|c|c||}
\hline Model & $\alpha$ & $\theta$ & $\mu$ & $\beta$ & $\lambda$ & $\boldsymbol{C D F}$ & References \\
\hline \hline$A W$ & - & - & - & - & 1 & $1-e^{-\left(\alpha x^{\theta}+\mu x^{\beta}\right)}$ & Xie and Lai (1995) \\
\hline$E M W$ & - & 1 & - & - & - & {$\left[1-e^{-\left(\alpha x+\mu x^{\beta}\right)}\right]^{\lambda}$} & Elbatal (2011) \\
\hline$M W$ & - & 1 & - & - & 1 & $1-e^{-\left(\alpha x+\mu x^{\beta}\right)}$ & Sarhan and Zaindin (2009) \\
\hline$E W$ & 0 & - & - & - & - & {$\left[1-e^{-\mu x^{\beta}}\right]^{\lambda}$} & Mudholkar and Srivastava (1993) \\
\hline$G E_{x}\left(E E_{x}\right)$ & - & 1 & 0 & - & - & {$\left[1-e^{-\alpha x}\right]^{\lambda}$} & Gupta and Kundu (1999) \\
\hline$G R$ & 0 & - & - & 2 & - & {$\left[1-e^{\left.-\mu x^{2}\right)}\right]^{\lambda}$} & Kundu and Raqab (2005) \\
\hline$G L F R$ & - & 1 & - & 2 & - & {$\left[1-e^{-\left(\alpha x+\mu x^{2}\right)}\right]^{\lambda}$} & Sarhan and Kundu (2009) \\
\hline$L F R$ & - & 1 & - & 2 & 1 & $1-e^{-\left(\alpha x+\mu x^{2}\right)}$ & Bain (1974) \\
\hline$W$ & 0 & - & - & - & 1 & $1-e^{-\mu x^{\beta}}$ & Weibull (1951) \\
\hline$E_{x}$ & - & 1 & 0 & - & 1 & $1-e^{-\alpha x}$ & Bain (1974) \\
\hline$R$ & 0 & - & - & 2 & 1 & $1-e^{-\mu x^{2}}$ & Bain (1974) \\
\hline
\end{tabular}

\section{Statistical Properties}

In this section we studied the shapes, statistical properties, specifically moments, incomplete moment, and moment generating function of the $(E A W)$ distribution.

\subsection{Shapes}

We provide the shapes of the $E A W$ density function (4) and the shapes of the $E A W$ failure rate function (6). After some mathematical proccess, the limit of the pdf (4) as $x$ approaches to 0 , is

$$
\lim _{x \rightarrow 0} f(x)= \begin{cases}0, & \lambda>1, \\ \infty, & 0<\lambda<1 \\ \infty, & 0<\theta<\beta<1 \text { or } 0<\beta<\theta<1, \lambda=1, \\ \infty, & \theta>1, \beta<1 \text { or } \theta<1, \beta>1, \lambda=1, \\ 0, & 1<\theta<\beta<\infty \text { or } 1<\beta<\theta<\infty, \lambda=1, \\ \alpha+\mu, & \theta=\beta=\lambda=1 .\end{cases}
$$

and the limit of the pdf (4) as $x$ approaches to $\infty$, is $\lim _{x \rightarrow \infty} f(x)=0$. 


\subsection{Moments}

In this section, the different moments of the exponentiated additive Weibull distribution can be obtained using the $r^{t h}$ moment $\mu_{r}^{\prime}=E\left(X^{r}\right)$ and the moment generating function, $M(t)=e^{t X}$.

Theorem 1 The $r^{\text {th }}$ moment of $E A W$ distribution, $r=1,2, \ldots$ is given by

$$
\mu_{r}^{\prime}=\lambda \sum_{j, k=0}^{\infty}\left(\begin{array}{c}
\lambda-1 \\
j
\end{array}\right)(-1)^{j+k} \frac{[\mu(j+1)]^{k}}{k !}\left[\frac{\alpha \Gamma\left(\frac{r+\beta k}{\theta}+1\right)}{[\alpha(j+1)]^{\frac{r+\beta k}{\theta}+1}}+\frac{\mu \beta \Gamma\left(\frac{r+\beta(k+1)}{\theta}\right)}{\theta[\alpha(j+1)]^{\frac{r+\beta(k+1)}{\theta}}}\right]
$$

Proof We start with the well known definition of the $r^{\text {th }}$ moment of the random variable $X$ with probability density function $f(x)$ given by

$$
\mu_{r}^{\prime}=\int_{0}^{\infty} x^{r} f(x, \alpha, \beta, \lambda, \theta) d x
$$

Substituting from (4) into the above relation, we get

$$
\mu_{r}^{\prime}=\lambda \int_{0}^{\infty} x^{r}\left(\alpha \theta x^{\theta-1}+\mu \beta x^{\beta-1}\right) e^{-\left(\alpha x^{\theta}+\mu x^{\beta}\right)}\left[1-e^{-\left(\alpha x^{\theta}+\mu x^{\beta}\right)}\right]^{\lambda-1} d x,
$$

since $0<e^{-\left(\alpha x^{\theta}+\mu x^{\beta}\right)}<1$ for $x>0$, then by using the binomial series expansion of $\left[1-e^{-\left(\alpha x^{\theta}+\mu x^{\beta}\right)}\right]^{\lambda-1}$ is given by

$$
\left[1-e^{-\left(\alpha x^{\theta}+\mu x^{\beta}\right)}\right]^{\lambda-1}=\sum_{j=0}^{\infty}\left(\begin{array}{c}
\lambda-1 \\
j
\end{array}\right)(-1)^{j} e^{-j\left(\alpha x^{\theta}+\mu x^{\beta}\right)},
$$

we get

$\mu_{r}^{\prime}=\lambda \sum_{j=0}^{\infty}\left(\begin{array}{c}\lambda-1 \\ j\end{array}\right)(-1)^{j} \int_{0}^{\infty}\left(\alpha \theta x^{r+\theta-1}+\mu \beta x^{r+\beta-1}\right) e^{-(j+1)\left(\alpha x^{\theta}+\mu x^{\beta}\right)} d x$,

but the series expansion of $e^{-(j+1) \mu x^{\beta}}$ is given by

$$
e^{-(j+1) \mu x^{\beta}}=\sum_{k=0}^{\infty} \frac{\left[-\mu(j+1) x^{\beta}\right]^{k}}{k !}
$$

substituting from (12) into (11), we get

$$
\mu_{r}^{\prime}=C_{j, k} \int_{0}^{\infty}\left(\alpha \theta x^{r+\beta k+\theta-1}+\mu \beta x^{r+\beta(k+1)-1}\right) e^{-(j+1) \alpha x^{\theta}} d x,
$$

where

$$
C_{j, k}=\lambda \sum_{j, k=0}^{\infty}\left(\begin{array}{c}
\lambda-1 \\
j
\end{array}\right)(-1)^{j+k} \frac{[\mu(j+1)]^{k}}{k !},
$$

setting $t=(j+1) \alpha x^{\theta}$, after some algebra, the integral in (13) can be computed as follows

$$
\mu_{r}^{\prime}=C_{j, k}\left[\frac{\alpha \Gamma\left(\frac{r+\beta k}{\theta}+1\right)}{[\alpha(j+1)]^{\frac{r+\beta k}{\theta}+1}}+\frac{\mu \beta \Gamma\left(\frac{r+\beta(k+1)}{\theta}\right)}{\theta[\alpha(j+1)]^{\frac{r+\beta(k+1)}{\theta}}}\right],
$$

which completes the proof. 
The central moments $\mu_{r}$ and cumulants $\kappa_{r}$ of the $E A W$ distribution can be determined from expression (8) as $\mu_{r}=\sum_{m=0}^{r}\left(\begin{array}{c}r \\ m\end{array}\right)(-1)^{m}\left(\mu_{1}^{\prime}\right)^{m} \mu_{r-m}^{\prime}$ and $\kappa_{r}=\mu_{r}^{\prime}-$ $\sum_{m=1}^{r-1}\left(\begin{array}{c}r-1 \\ m-1\end{array}\right) \kappa_{m} \mu_{r-m}^{\prime}$, respectively, where $\kappa_{1}=\mu_{1}^{\prime}, \kappa_{2}=\mu_{2}^{\prime}-\left(\mu_{1}^{\prime}\right)^{2}, \kappa_{3}=\mu_{3}^{\prime}-3 \mu_{2}^{\prime}$ $\mu_{1}^{\prime}+2\left(\mu_{1}^{\prime}\right)^{3}$, and $\kappa_{4}=\mu_{4}^{\prime}-4 \mu_{1}^{\prime} \mu_{3}^{\prime}-3\left(\mu_{2}^{\prime}\right)^{2}+12 \mu_{2}^{\prime}\left(\mu_{1}^{\prime}\right)^{2}-6\left(\mu_{1}^{\prime}\right)^{4}$, etc. Additionally, the skewness and kurtosis can be calculated from the third and fourth standardized cumulants in the forms $S K=\kappa_{3} / \sqrt{\kappa_{2}^{3}}$ and $K U=\kappa_{4} / \kappa_{2}^{2}$, respectively.

Theorem 2 The moment generating function of $E A W$ distribution is given by

$$
M_{X}(t)=\sum_{j, k, r=0}^{\infty} \frac{t^{r}}{r !} \lambda\left(\begin{array}{c}
\lambda-1 \\
j
\end{array}\right)(-1)^{j+k} \frac{[\mu(j+1)]^{k}}{k !}\left[\frac{\alpha \Gamma\left(\frac{r+\beta k}{\theta}+1\right)}{[\alpha(j+1)]^{\frac{r+\beta k}{\theta}+1}}+\frac{\mu \beta \Gamma\left(\frac{r+\beta(k+1)}{\theta}\right)}{\theta[\alpha(j+1)]^{\frac{r+\beta(k+1)}{\theta}}}\right] .
$$

Proof We start with the well known definition of the moment generating function given by $M_{X}(t)=E\left(e^{t X}\right)=\int_{0}^{\infty} e^{t x} f_{E A W}(x, \boldsymbol{\phi}) d x$, since $\sum_{r=0}^{\infty} \frac{t^{r}}{r !} x^{r} f(x)$ converges and each term is integrable for all $t$ close to 0 , then we can rewrite the moment generating function as $M_{X}(t)=\sum_{r=0}^{\infty} \frac{t^{r}}{r !} E\left(X^{r}\right)$ by replacing $E\left(X^{r}\right)$. Hence using (8) the MGF of EAW distribution is given by

$$
M_{X}(t)=\sum_{j, k, r=0}^{\infty} \frac{t^{r}}{r !} \lambda\left(\begin{array}{c}
\lambda-1 \\
j
\end{array}\right)(-1)^{j+k} \frac{[\mu(j+1)]^{k}}{k !}\left[\frac{\alpha \Gamma\left(\frac{r+\beta k}{\theta}+1\right)}{[\alpha(j+1)]^{\frac{r+\beta k}{\theta}+1}}+\frac{\mu \beta \Gamma\left(\frac{r+\beta(k+1)}{\theta}\right)}{\theta[\alpha(j+1)]^{\frac{r+\beta(k+1)}{\theta}}}\right] .
$$

which completes the proof.

Similarly, the characteristic function of the $E A W$ distribution becomes $\phi_{X}(t)=M_{X}(i t)$ where $i=\sqrt{-1}$ is the unit imaginary number.

\subsection{Conditional Moments}

The main application of the first incomplete moment refers to the Bonferroni and Lorenz curves. These curves are very useful in economics, reliability, demography, insurance and medicine. The answers to many important questions in economics require more than just knowing the mean of the distribution, but its shape as well. This is obvious not only in the study of econometrics but in other areas as well. For lifetime models, it is also of interest to find the conditional moments and the mean residual lifetime function. The conditional moments for $E A W$ distribution is given by

$$
\begin{aligned}
v_{s}=E\left(X^{s} \mid X>t\right) & =\int_{t}^{\infty} x^{s} f_{E A W}(x, \boldsymbol{\phi}) d x . \\
& =C_{j, k} \int_{t}^{\infty}\left(\alpha \theta x^{s+\beta k+\theta-1}+\mu \beta x^{s+\beta(k+1)-1}\right) e^{-(j+1) \alpha x^{\theta}} d x . \\
& =C_{j, k}\left[\frac{\alpha \Gamma\left(\frac{s+\beta k}{\theta}+1,(j+1) \alpha t^{\theta}\right)}{[\alpha(j+1)]^{\frac{s+\beta k}{\theta}+1}}+\frac{\mu \beta \Gamma\left(\frac{s+\beta(k+1)}{\theta},(j+1) \alpha t^{\theta}\right)}{\theta[\alpha(j+1)]^{\frac{s+\beta(k+1)}{\theta}}}\right] .
\end{aligned}
$$

where $\Gamma(s, t)=\int_{t}^{\infty} x^{s-1} e^{-x} d x$ is the upper incomplete gamma function. The mean residual lifetime function is given by

$$
\mu(t)=E(X \mid X>t)-t=C_{j, k}\left[\frac{\alpha \Gamma\left(\frac{\beta k+1}{\theta}+1,(j+1) \alpha t^{\theta}\right)}{[\alpha(j+1)]^{\frac{s+\beta k}{\theta}+1}}+\frac{\mu \beta \Gamma\left(\frac{\beta(k+1)+1}{\theta},(j+1) \alpha t^{\theta}\right)}{\theta[\alpha(j+1)]^{\frac{s+\beta(k+1)}{\theta}}}\right]-t .
$$




\subsection{Quantile Function}

The quantile function (qf) of $X$ is obtained by inverting (3), but there is no colsed form for the qf of $E A W$ distribtion. The $p^{\text {th }}$ quantail of the $E A W$ distribtion can be obtained by numerically solving the following equaiton for $x$

$$
\ln \left(1-p^{\frac{1}{\lambda}}\right)+\alpha x^{\theta}+\mu x^{\beta}=0 \text {. }
$$

One can simulate the $E A W$ random variable with $\phi=(\alpha, \theta, \mu, \beta, \lambda)^{T}$ by:

1. Generate $U \sim$ nuiform $(0,1)$.

2. Put $p=U$ in equation (18).

3. Solve equation (18) numerically for $x$.

The median can be calculated by putting $\boldsymbol{p}=\mathbf{0 . 5}$ in equation (18), and solving it numerically for $\boldsymbol{x}$.

\section{Residual life and Reversed Failure Rate Function}

Given that a component survives up to time $t \geq 0$, the residual life is the period beyond $\mathrm{t}$ until the time of failure and defined by the conditional random variable $X-t \mid X>t$. In reliability, it is well known that the mean residual life function and ratio of two consecutive moments of residual life determine the distribution uniquely (Gupta and Gupta, 1983). Therefore, we obtain the $r^{\text {th }}$ order moment of the residual lifetime can be obtained via the general formula

$$
\mu_{r}(t)=E\left((X-t)^{r} \mid X>t\right)=\frac{1}{\bar{F}(t)} \int_{t}^{\infty}(x-t)^{r} f(x, \phi) d x, r \geq 1 .
$$

Applying the binomial expansion of $(x-t)^{r}$ into the above formula, we get

$$
\begin{aligned}
& \mu_{r}(t)=\frac{1}{\bar{F}(t)} \sum_{d=0}^{r}(-t)^{d}\left(\begin{array}{l}
r \\
d
\end{array}\right) \int_{t}^{\infty} x^{r-d} f(x) d x . \\
& =\frac{C_{j, k}}{\bar{F}(t)} \sum_{d=0}^{r}(-t)^{d}\left(\begin{array}{l}
r \\
d
\end{array}\right) \int_{t}^{\infty}\left(\alpha \theta x^{r+\beta k+\theta-d-1}+\mu \beta x^{r+\beta(k+1)-d-1}\right) e^{-(j+1) \alpha x^{\theta}} d x . \\
& =\frac{C_{j, k}}{\bar{F}(t)} \sum_{d=0}^{r}(-t)^{d}\left(\begin{array}{l}
r \\
d
\end{array}\right)\left[\frac{\alpha \Gamma\left(\frac{r+\beta k-d}{\theta}+1,(j+1) \alpha t^{\theta}\right)}{[\alpha(j+1)]^{\frac{r+\beta k-d}{\theta}+1}}+\frac{\mu \beta \Gamma\left(\frac{r+\beta(k+1)-d}{\theta},(j+1) \alpha t^{\theta}\right)}{\theta[\alpha(j+1)]^{\frac{r+\beta(k+1)-d}{\theta}}}\right] .
\end{aligned}
$$

The mean residual life (MRL) of the $E A W$ distribution is given by

$$
\mu(t)=\frac{C_{j, k}}{\bar{F}(t)}\left[\frac{\alpha \Gamma\left(\frac{\beta k+1}{\theta}+1,(j+1) \alpha t^{\theta}\right)}{[\alpha(j+1)]^{\frac{\beta k+1}{\theta}+1}}+\frac{\mu \beta \Gamma\left(\frac{\beta(k+1)+1}{\theta},(j+1) \alpha t^{\theta}\right)}{\theta[\alpha(j+1)]^{\frac{\beta(k+1)+1}{\theta}}}\right]-t .
$$

The variance of the residual life of the $E A W$ distribution can be obtained easily by using $\mu_{2}(t)$ and $\mu(t)$. 
On the other hand, we analogously discuss the reversed residual life and some of its properties. The reversed residual life can be defined as the conditional random variable $t-X \mid X \leq t$ which denotes the time elapsed from the failure of a component given that its life is less than or equal to $t$. This random variable may also be called the inactivity time (or time since failure); for more details you may see (Kundu and Nanda, 2010).

Also, in reliability, the mean reversed residual life and ratio of two consecutive moments of reversed residual life characterize the distribution uniquely. The reversed failure (or reversed hazard) rate function is given by Equation (7). The $r^{\text {th }}$ order moment of the reversed residual life can be obtained by the well known formula

$$
m_{r}(t)=E\left((t-X)^{r} \mid X \leq t\right)=\frac{1}{F(t)} \int_{0}^{t}(t-x)^{r} f(x, \phi) d x, r \geq 1 .
$$

Applying the binomial expansion of $(t-x)^{r}$ into the above formula gives

$$
\begin{aligned}
m_{r}(t) & =\frac{w_{i, j, k}}{F(t)} \sum_{d=0}^{r}(-t)^{d}\left(\begin{array}{l}
r \\
d
\end{array}\right) \int_{0}^{t}\left(\alpha \theta x^{r+\beta k+\theta-d-1}+\mu \beta x^{r+\beta(k+1)-d-1}\right) e^{-(j+1) \alpha x^{\theta}} d x . \\
& =\frac{C_{j, k}}{F(t)} \sum_{d=0}^{r}(-t)^{d}\left(\begin{array}{l}
r \\
d
\end{array}\right)\left[\frac{\alpha \zeta\left(\frac{r+\beta k-d}{\theta}+1,(j+1) \alpha t^{\theta}\right)}{[\alpha(j+1)]^{\frac{r+\beta k-d}{\theta}+1}}+\frac{\mu \beta \zeta\left(\frac{r+\beta(k+1)-d}{\theta},(j+1) \alpha t^{\theta}\right)}{\theta[\alpha(j+1)]^{\frac{r+\beta(k+1)-d}{\theta}}}\right] .
\end{aligned}
$$

where $\zeta(s, t)=\int_{0}^{t} x^{s-1} e^{-x} d x$ is the lower incomplete gamma function. Thus, the mean of the reversed residual life of the $E A W$ distribution is given by

$m(t)=t-\frac{C_{j, k}}{F(t)}\left[\frac{\alpha \zeta\left(\frac{\beta k+1}{\theta}+1,(j+1) \alpha t^{\theta}\right)}{[\alpha(j+1)]^{\frac{\beta k+1}{\theta}+1}}+\frac{\mu \beta \zeta\left(\frac{\beta(k+1)+1}{\theta},(j+1) \alpha t^{\theta}\right)}{\theta[\alpha(j+1)]^{\frac{\beta(k+1)+1}{\theta}}}\right]$.

Using $m(t)$ and $m_{2}(t)$ one can obtain the variance and the coefficient of variation of the reversed residual life of the $E A W$ distribution.

\subsection{Bonferroni and Lorenz Curves}

In this subsection we proposed the Bonferroni and Lorenz Curves. The Bonferroni and Lorenz curves (Bonferroni, 1930) and the Bonferroni and Gini indices have applications not only in economics to study income and poverty, but also in other fields like reliability, demography, insurance and medicine. The Bonferroni and Lorenz curves are defined by

$$
B(p)=\frac{1}{p \mu} \int_{0}^{q} x f(x) d x=\frac{C_{j, k}}{p \mu}\left[\frac{\alpha \zeta\left(\frac{\beta k+1}{\theta}+1,(j+1) q t^{\theta}\right)}{[\alpha(j+1)]^{\frac{\beta k+1}{\theta}+1}}+\frac{\mu \beta \zeta\left(\frac{\beta(k+1)+1}{\theta},(j+1) q t^{\theta}\right)}{\theta[\alpha(j+1)]^{\frac{\beta(k+1)+1}{\theta}}}\right]
$$

and

$$
L(p)=\frac{1}{\mu} \int_{0}^{q} x f(x) d x=\frac{C_{j, k}}{\mu}\left[\frac{\alpha \zeta\left(\frac{\beta k+1}{\theta}+1,(j+1) q t^{\theta}\right)}{[\alpha(j+1)]^{\frac{\beta k+1}{\theta}+1}}+\frac{\mu \beta \zeta\left(\frac{\beta(k+1)+1}{\theta},(j+1) q t^{\theta}\right)}{\theta[\alpha(j+1)]^{\frac{\beta(k+1)+1}{\theta}}}\right] .
$$

\subsection{Mean deviation}

In statistics, mean deviation about the mean and mean deviation about the median measure the amount of scatter in a population. For random variable $X$ with pdf $f(x)$, 
distribution function $F(x)$, mean $\mu=E(X)$ and $M=$ Median $(X)$, the mean deviation about the mean and mean deviation about the median, are defined by

and

$$
\delta_{1}(x)=\int_{0}^{\infty}|x-\mu| f(x) d x=2 \mu F(\mu)-2 \mu+2 \int_{\mu}^{\infty} x f(x) d x
$$

$$
\delta_{2}(x)=\int_{0}^{\infty}|x-M| f(x) d x=2 M F(M)-M-\mu+2 \int_{M}^{\infty} x f(x) d x
$$

respectively, if $X$ is $E A W$ random variable then

$$
\int_{\mu}^{\infty} x f(x) d x=C_{j, k}\left[\frac{\alpha \Gamma\left(\frac{\beta k+1}{\theta}+1,(j+1) \mu t^{\theta}\right)}{[\alpha(j+1)]^{\frac{\beta k+1}{\theta}+1}}+\frac{\mu \beta \Gamma\left(\frac{\beta(k+1)+1}{\theta},(j+1) \mu t^{\theta}\right)}{\theta[\alpha(j+1)]^{\frac{\beta(k+1)+1}{\theta}}}\right]
$$

and

$$
\int_{M}^{\infty} x f(x) d x=C_{j, k}\left[\frac{\alpha \Gamma\left(\frac{\beta k+1}{\theta}+1,(j+1) M t^{\theta}\right)}{[\alpha(j+1)]^{\frac{\beta k+1}{\theta}+1}}+\frac{\mu \beta \Gamma\left(\frac{\beta(k+1)+1}{\theta},(j+1) M t^{\theta}\right)}{\theta[\alpha(j+1)]^{\frac{\beta(k+1)+1}{\theta}}}\right]
$$

so that

$$
\delta_{1}(x)=2 \mu F(\mu)+2 C_{j, k}\left[\frac{\alpha \Gamma\left(\frac{\beta k+1}{\theta}+1,(j+1) \mu t^{\theta}\right)}{[\alpha(j+1)]^{\frac{\beta k+1}{\theta}+1}}+\frac{\mu \beta \Gamma\left(\frac{\beta(k+1)+1}{\theta},(j+1) \mu t^{\theta}\right)}{\theta[\alpha(j+1)]^{\frac{\beta(k+1)+1}{\theta}}}\right]-2 \mu
$$

and

$$
\delta_{2}(x)=-\mu+2 C_{j, k}\left[\frac{\alpha \Gamma\left(\frac{\beta k+1}{\theta}+1,(j+1) M t^{\theta}\right)}{[\alpha(j+1)]^{\frac{\beta k+1}{\theta}+1}}+\frac{\mu \beta \Gamma\left(\frac{\beta(k+1)+1}{\theta},(j+1) M t^{\theta}\right)}{\theta[\alpha(j+1)]^{\frac{\beta(k+1)+1}{\theta}}}\right] .
$$

\section{Maximum Likelihood Estimation}

Statistical inference can be carried out in three different ways: point estimation, interval estimation and hypothesis testing. Several approaches for parameter point estimation were proposed in the literature but the maximum likelihood method is the most commonly employed. The MLES enjoy desirable properties and can be used when constructing confidence intervals and regions and also in test statistics. Here, we determine the maximum likelihood estimates (MLES) of the parameters of the $E A W$ distribution from complete samples only. Let $x_{1}, \ldots, x_{n}$ be a random sample of size $n$ from the $E A W$ distribution given by (4). Let $\phi=(\alpha, \theta, \mu, \beta, \lambda)^{T}$ be $5 \times 1$ vector of parameters. The total $\log$-likelihood function for $\boldsymbol{\phi}$ is given by

$$
\begin{aligned}
\ell_{n}=\ell_{n}(\boldsymbol{\phi})=n \log \lambda & +\sum_{i=1}^{n} \log \left(\alpha \theta x_{i}^{\theta-1}+\mu \beta x_{i}^{\beta-1}\right)-\alpha \sum_{i=1}^{n} x_{i}^{\theta}-\mu \sum_{i=1}^{n} x_{i}^{\beta} \\
& +(\lambda-1) \sum_{i=1}^{n} \log \left[1-e^{-\alpha x_{i}^{\theta}-\mu x_{i}^{\beta}}\right] .
\end{aligned}
$$

The log-likelihood can be maximized either directly by using the SAS program or $R$ language (2018) or by solving the nonlinear likelihood equations obtained by differentiating (26). The associated components of the score function $U_{n}(\boldsymbol{\phi})=$ $\left[\frac{\partial \ell_{n}}{\partial \alpha}, \frac{\partial \ell_{n}}{\partial \theta}, \frac{\partial \ell_{n}}{\partial \mu}, \frac{\partial \ell_{n}}{\partial \beta}, \frac{\partial \ell_{n}}{\partial \lambda}\right]^{T}$ are 


$$
\begin{aligned}
& \frac{\partial \ell_{n}}{\partial \alpha}=\sum_{i=1}^{n} \frac{\theta x_{i}^{\theta-1}}{\alpha \theta x_{i}^{\theta-1}+\mu \beta x_{i}^{\beta-1}}-\sum_{i=1}^{n} x_{i}^{\theta}+(\lambda-1) \sum_{i=1}^{n} \frac{x_{i}^{\theta} e^{-\alpha x^{\theta}-\mu x^{\beta}}}{1-e^{-\alpha x^{\theta}-\mu x^{\beta}}}, \\
& \frac{\partial \ell_{n}}{\partial \theta}=\alpha \sum_{i=1}^{n} \frac{x_{i}^{\theta-1}\left(\theta \ln \left(x_{i}\right)+1\right)}{\alpha \theta x_{i}^{\theta-1}+\mu \beta x_{i}^{\beta-1}}-\alpha \sum_{i=1}^{n} x_{i}^{\theta} \ln \left(x_{i}\right)+\alpha(\lambda-1) \sum_{i=1}^{n} \frac{x_{i}^{\theta} \ln \left(x_{i}\right) e^{-\alpha x_{i}^{\theta}-\mu x_{i}^{\beta}}}{1-e^{-\alpha x_{i}^{\theta}-\mu x_{i}^{\beta}}}, \\
& \frac{\partial \ell_{n}}{\partial \mu}=\sum_{i=1}^{n} \frac{\beta x_{i}^{\beta-1}}{\alpha \theta x_{i}^{\theta-1}+\mu \beta x_{i}^{\beta-1}}-\sum_{i=1}^{n} x_{i}^{\beta}+(\lambda-1) \sum_{i=1}^{n} \frac{x_{i}^{\beta} e^{-\alpha x_{i}^{\theta}-\mu x_{i}^{\beta}}}{1-e^{-\alpha x_{i}^{\theta}-\mu x_{i}^{\beta}}}, \\
& \frac{\partial \ell_{n}}{\partial \beta}=\mu \sum_{i=1}^{n} \frac{x_{i}^{\beta-1}\left(\beta \ln \left(x_{i}\right)+1\right)}{\alpha \theta x_{i}^{\theta-1}+\mu \beta x_{i}^{\beta-1}}-\mu \sum_{i=1}^{n} x_{i}^{\beta} \ln \left(x_{i}\right)+\mu(\lambda-1) \sum_{i=1}^{n} \frac{x_{i}^{\beta} \ln \left(x_{i}\right) e^{-\alpha x_{i}^{\theta}-\mu x_{i}^{\beta}}}{1-e^{-\alpha x_{i}^{\theta}-\mu x_{i}^{\beta}}} \\
& \text { and } \\
& \frac{\partial \ell_{n}}{\partial \lambda}=\frac{n}{\lambda}+\sum_{i=1}^{n} \log \left[1-e^{\left.-\alpha x_{i}^{\theta}-\mu x_{i}^{\beta}\right] .}\right.
\end{aligned}
$$

The maximum likelihood estimation $(M L E)$ of $\boldsymbol{\phi}$, say $\widehat{\boldsymbol{\phi}}$, is obtained by solving the nonlinear system $U_{n}(\boldsymbol{\phi})=0$. These equations cannot be solved analytically, and statistical software can be used to solve them numerically via iterative methods. We can use iterative techniques such as a Newton-Raphson type algorithm to obtain the estimate $\widehat{\boldsymbol{\phi}}$. For interval estimation and hypothesis tests on the model parameters, we require the information matrix. The $5 \times 5$ observed information matrix is given by

$$
I_{n}(\boldsymbol{\phi})=-\left(\begin{array}{lllll}
I_{\alpha \alpha} & I_{\alpha \theta} & I_{\alpha \mu} & I_{\alpha \beta} & I_{\alpha \lambda} \\
I_{\theta \alpha} & I_{\theta \theta} & I_{\theta \mu} & I_{\theta \beta} & I_{\theta \lambda} \\
I_{\mu \alpha} & I_{\mu \theta} & I_{\mu \mu} & I_{\mu \beta} & I_{\mu \lambda} \\
I_{\beta \alpha} & I_{\beta \theta} & I_{\beta \mu} & I_{\beta \beta} & I_{\beta \lambda} \\
I_{\lambda \alpha} & I_{\lambda \theta} & I_{\lambda \mu} & I_{\lambda \beta} & I_{\lambda \lambda}
\end{array}\right)
$$

whose elements are given in the Appendix. Applying the usual large sample approximation, $M L E$ of $\boldsymbol{\phi}$, i.e $\widehat{\boldsymbol{\phi}}$ can be treated as being approximately $N_{5}\left(\boldsymbol{\phi}, J_{n}(\boldsymbol{\phi})^{-1}\right)$, where $J_{n}(\boldsymbol{\phi})=E\left[I_{n}(\boldsymbol{\phi})\right]$. Under conditions that are fulfilled for parameters in the interior of the parameter space but not on the boundary, the asymptotic distribution of $\sqrt{n}(\widehat{\boldsymbol{\phi}}-\boldsymbol{\phi})$ is $N_{5}\left(0, J(\boldsymbol{\phi})^{-1}\right)$, where $J(\boldsymbol{\phi})=\lim _{n \rightarrow \infty} n^{-1} I_{n}(\boldsymbol{\phi})$ is the unit information matrix. This asymptotic behavior remains valid if $J(\phi)$ is replaced by the average sample information matrix evaluated at $\widehat{\boldsymbol{\phi}}$, say $n^{-1} I_{n}(\widehat{\boldsymbol{\phi}})$. The estimated asymptotic multivariate normal $N_{5}\left(\boldsymbol{\phi}, I_{n}(\widehat{\boldsymbol{\phi}})^{-1}\right)$ distribution of $\widehat{\boldsymbol{\phi}}$ can be used to construct approximate confidence intervals for the parameters and for the hazard rate and survival functions. An $100(1-\gamma) \%$ asymptotic confidence interval for each parameter $\boldsymbol{\phi}_{r}$ is given by

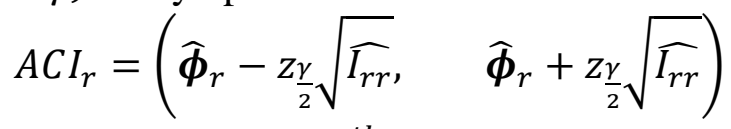

where $z_{\gamma}$ is the upper $100 \gamma^{\text {th }}$ percentile of the standard normal distribution.

\section{Simulation Study}

To test the validating of the theoretical results in Section 5, we produced simulations by $\mathrm{R}$ statistical package, by generating 2,000 samples of sizes $n=\{50,65,80,95,110,125$, $140,155,170,185,200,215\}$ from the $E A W$ distribution by (18), with true parametric values of $\phi: \quad(\alpha=1.5, \theta=5.0, \mu=0.5, \beta=0.8, \lambda=2.5)^{T}$ and $(\alpha=1.5, \theta=3.0, \mu=$ 
$0.5, \beta=0.8, \lambda=1.0)^{T}$. The average of absolute value of biases, $|\operatorname{Bias}(\widehat{\boldsymbol{\phi}})|=$ $\frac{1}{N} \sum_{i=1}^{n}|\widehat{\boldsymbol{\phi}}-\boldsymbol{\phi}|$, and the mean square error of the estimates, $\operatorname{MSE}(\widehat{\boldsymbol{\phi}})=\frac{1}{N} \sum_{i=1}^{n}(\widehat{\boldsymbol{\phi}}-\boldsymbol{\phi})^{2}$, are computed.

These results are reported in Table 2. We can see that the values of $|\operatorname{Bias}(\widehat{\boldsymbol{\phi}})|$ and $\operatorname{MSE}(\widehat{\boldsymbol{\phi}})$ decrease as sample size increases.

Table 2: Average values of $|\operatorname{Bias}(\widehat{\boldsymbol{\phi}})|$ and the corresponding $\operatorname{MSES}(\widehat{\boldsymbol{\phi}})$

\begin{tabular}{|c|c|c|c|c|c|c|c|c|c|c|}
\hline \multicolumn{11}{|c|}{$\phi=(\alpha=1.5, \theta=5.0, \mu=0.5, \beta=0.8, \lambda=2.5)^{T}$} \\
\hline \multirow[b]{2}{*}{$n$} & \multicolumn{5}{|c|}{$|\operatorname{Bias}(\widehat{\boldsymbol{\phi}})|$} & \multicolumn{5}{|c|}{$\operatorname{MSE}(\widehat{\boldsymbol{\phi}})$} \\
\hline & $\hat{\alpha}$ & $\widehat{\theta}$ & $\hat{\mu}$ & $\hat{\beta}$ & $\hat{\lambda}$ & $\hat{\alpha}$ & $\hat{\theta}$ & $\hat{\mu}$ & $\hat{\beta}$ & $\hat{\lambda}$ \\
\hline 50 & 0.5761 & 29814 & 51799 & 0.86305 & 0.30088 & 55355 & 7.53188 & 0.49802 & 1.32654 & 14576 \\
\hline 65 & 0203 & 09122 & 0.43974 & 0.73273 & 0.25280 & 0.42795 & 52.16778 & 0.39603 & 1.04794 & 10306 \\
\hline 80 & 3713 & 52463 & 0.39121 & 0.65407 & 0.22458 & 0.36226 & 37.76243 & 0.32699 & 0.86033 & 07982 \\
\hline 95 & 1 & 13125 & 0.36282 & 0.61452 & 0.21061 & 0.31778 & 28.84269 & 0.28864 & 0.77003 & 7079 \\
\hline 110 & 4 & 49443 & 0.30870 & \begin{tabular}{|l|}
0.52898 \\
\end{tabular} & \begin{tabular}{|l|}
0.19748 \\
\end{tabular} & 0.23596 & 13.85096 & \begin{tabular}{|l|}
0.21070 \\
\end{tabular} & 0.57212 & 6151 \\
\hline 125 & & 1.44014 & 0.29469 & 0.50771 & 0.18231 & 0.22228 & 14.87032 & 0.19975 & 0.54232 & 5251 \\
\hline 140 & & 1.11273 & 0.25848 & 0.45386 & 0.17255 & & 7.81345 & 0.15810 & 0.43699 & 4803 \\
\hline 155 & & 00 & & \begin{tabular}{|l|}
0.40887 \\
\end{tabular} & 0.1 & & 4.48580 & \begin{tabular}{|l|}
0.12575 \\
\end{tabular} & 0.35057 & 4033 \\
\hline 170 & & 40 & & 0.40200 & & & 4.40740 & \begin{tabular}{|l|}
0.11806 \\
\end{tabular} & 0.34442 & 3642 \\
\hline 185 & & 073 & & \begin{tabular}{|l|}
0.37514 \\
\end{tabular} & 079 & & 2.48610 & \begin{tabular}{|l|}
0.09841 \\
\end{tabular} & 0.28683 & 3648 \\
\hline 200 & & 0781 & & \begin{tabular}{|l|}
0.37323 \\
\end{tabular} & 123 & & 3.27028 & \begin{tabular}{|l|}
0.10093 \\
\end{tabular} & 0.28895 & 0.03175 \\
\hline 215 & & 64552 & 0.18912 & 0.34422 & 0.13317 & 0.09203 & 1.98047 & 0.07660 & 0.23179 & 0.02881 \\
\hline \multicolumn{11}{|c|}{$\phi=(\alpha=1.5, \theta=3.0, \mu=0.5, \beta=0.8, \lambda=1.0)^{T}$} \\
\hline & \multicolumn{5}{|c|}{$|\operatorname{Bias}(\widehat{\boldsymbol{\phi}})|$} & \multicolumn{5}{|c|}{$\operatorname{MSE}(\widehat{\boldsymbol{\phi}})$} \\
\hline$n$ & $\hat{\alpha}$ & $\hat{\theta}$ & $\hat{\mu}$ & $\hat{\beta}$ & $\hat{\lambda}$ & $\hat{\alpha}$ & $\hat{\theta}$ & $\hat{\mu}$ & $\hat{\beta}$ & $\hat{\lambda}$ \\
\hline 50 & 0.472 & 351 & 733 & 0.2904 & 0.12029 & 38673 & 44.14962 & 27557 & 14808 & 0272 \\
\hline 65 & & 1 & & 0.24059 & 0. & 0.2 & 15.98097 & 662 & & 247 \\
\hline 80 & & & & 0.2 & & & 6.30872 & 462 & & \\
\hline 95 & & & & 0.19913 & 0.0 & & 3.57361 & 048 & 0.0 & \\
\hline 110 & & & & 0.18095 & 0.0 & & 3.38734 & 583 & 0.0 & 222 \\
\hline 125 & & & & 0.17205 & & & 1.77998 & 061 & & \\
\hline 140 & & & & 0.15689 & 0.0 & & 1.25309 & 0.07441 & & 265 \\
\hline 155 & & & & 0.15191 & & & 0.81173 & \begin{tabular}{|l|}
0.07087 \\
\end{tabular} & 0.04 & \\
\hline 170 & & & 0.1 & \begin{tabular}{|l|}
0.13991 \\
\end{tabular} & \begin{tabular}{|l|}
0.07958 \\
\end{tabular} & & 0.62951 & \begin{tabular}{|l|}
0.05631 \\
\end{tabular} & & 0.02445 \\
\hline 18 & & & & 0.13 & & & 0.39 & \begin{tabular}{|l|}
0.05227 \\
\end{tabular} & & \\
\hline 200 & 0.20041 & 0.38007 & 0.15828 & 0.13092 & 0.07134 & 0.06799 & 0.31900 & \begin{tabular}{|l|}
0.04586 \\
\end{tabular} & 0.02849 & 0.01999 \\
\hline 215 & 0.1949 & 0.35905 & 0.1501 & 0.12247 & 0.07823 & 0.06487 & 0.29628 & 0.04112 & 0.02526 & 0.02834 \\
\hline
\end{tabular}

\section{Application}

In this section we illustrate the superiority of the new distribution as compared to some of its submodels and also to the alternative distributions. For the data set, the estimates of the parameters of the distributions and information criterion statistics are calculated. Here, we provide application to real data in order to illustrate the potentiality of the $E A W$ model. The measures of goodness-of-fit including the log-likelihood function evaluated at the MLEs, Cramer-von Mises $\left(W^{*}\right)$, Anderson-Darling $\left(A^{*}\right)$ and Kolmogorov-Smirnov $(K-S)$ statistic with its $p$-value are calculated to compare the fitted models. In general, 
the smallest the values of $W^{*}, A^{*}$ and $K-S$ statistics, and the largest $K-S p$-value, is considered the best fit to the data. The required computations are carried out using the $R$ software.

The data used in this research is corresponding to remission times (in months) of a random sample of 128 bladder cancer patients given in Lee and Wang (2003). The data is given as follows:

$0.08,0.20,0.40,0.50,0.51,0.81,0.90,1.05,1.19,1.26,1.35,1.40,1.46,1.76,2.02,2.02$, $2.07,2.09,2.23,2.26,2.46,2.54,2.62,2.64,2.69,2.69,2.75,2.83,2.87,3.02,3.25$, 3.31 , 3.36, 3.36, 3.48, 3.52, 3.57, 3.64, 3.70, 3.82, 3.88, 4.18, 4.23, 4.26, 4.33, 4.34, 4.40, $4.50,4.51,4.87,4.98,5.06,5.09,5.17,5.32,5.32,5.34,5.41,5.41,5.49,5.62,5.71,5.85$, $6.25,6.54,6.76,6.93,6.94,6.97,7.09,7.26,7.28,7.32,7.39,7.59,7.62,7.63,7.66,7.87$, $7.93,8.26,8.37,8.53,8.65,8.66,9.02,9.22,9.47,9.74,10.06,10.34,10.66,10.75,11.25$, $11.64,11.79,11.98,12.02,12.03,12.07,12.63,13.11,13.29,13.80,14.24,14.76,14.77$, $14.83,15.96,16.62,17.12,17.14,17.36,18.10,19.13,20.28,21.73,22.69,23.63,25.74$, $25.82,26.31,32.15,34.26,36.66,43.01,46.12,79.05$.

Table 3: The statistics: $-2 \ell_{128}(\widehat{\phi}), W^{*}$ and $A^{*}$ for the bladder cancer data

\begin{tabular}{|l||c|c|c|c|c||}
\hline Model & $-2 \ell_{128}(\widehat{\boldsymbol{\phi}})$ & $W^{*}$ & $A^{*}$ & $K-S$ & $p$-value \\
\hline \hline $\boldsymbol{E A W}$ & $\mathbf{8 1 9 . 7 4 6}$ & $\mathbf{0 . 0 2 3 5}$ & $\mathbf{0 . 1 5 2 3}$ & $\mathbf{0 . 0 3 4 9}$ & $\mathbf{0 . 9 9 7 6}$ \\
\hline $\boldsymbol{K} \boldsymbol{W}-\boldsymbol{M W}$ & 821.504 & 0.0457 & 0.3007 & 0.0456 & 0.9530 \\
\hline $\boldsymbol{M} \boldsymbol{W}$ & 828.175 & 0.1314 & 0.7864 & 0.0700 & 0.5575 \\
\hline $\boldsymbol{W}$ & 828.174 & 0.1314 & 0.7865 & 0.0700 & 0.5570 \\
\hline $\boldsymbol{E} \boldsymbol{E}_{\boldsymbol{x}}$ & 826.155 & 0.1122 & 0.6741 & 0.0725 & 0.5113 \\
\hline $\boldsymbol{E}_{\boldsymbol{x}}$ & 828.684 & 0.1193 & 0.7160 & 0.0846 & 0.3184 \\
\hline $\boldsymbol{E} \boldsymbol{W}$ & 821.360 & 0.0437 & 0.2885 & 0.0450 & 0.9576 \\
\hline $\boldsymbol{R}$ & 982.531 & 0.4664 & 2.7300 & 0.3521 & 0.0000 \\
\hline
\end{tabular}

Table 4: $M L E s$ and their standard errors (in parentheses) for the bladder cancer data

\begin{tabular}{|c|c|c|c|c|c|}
\hline Model & \multicolumn{5}{|c|}{ ML Estimates } \\
\hline$E A W$ & $\begin{array}{c}\hat{\alpha}=0.8101 \\
(0.5901)\end{array}$ & $\begin{array}{c}\hat{\theta}=0.5299 \\
(0.1483)\end{array}$ & $\begin{array}{c}\hat{\mu}=2.1056 \\
(4.1206)\end{array}$ & $\begin{array}{c}\hat{\beta}=0.0157 \\
(0.0862)\end{array}$ & $\begin{array}{c}\hat{\lambda}=51.7587 \\
(222.2643)\end{array}$ \\
\hline$K w-M W$ & $\begin{array}{c}\hat{a}=3.9883 \\
(4.3741)\end{array}$ & $\begin{array}{c}\hat{b}=2.0482 \\
(5.7773)\end{array}$ & $\begin{array}{c}\hat{\alpha}=0.5346 \\
(0.5122)\end{array}$ & $\begin{array}{c}\hat{\gamma}=0.4842 \\
(0.4217)\end{array}$ & $\begin{array}{c}\hat{\lambda}=0.0010 \\
(0.0067)\end{array}$ \\
\hline$M W$ & $\begin{array}{c}\hat{\alpha}=0.0010 \\
(0.3076)\end{array}$ & $\begin{array}{c}\hat{\mu}=0.0929 \\
(0.2946)\end{array}$ & $\begin{array}{c}\hat{\beta}=1.0481 \\
(0.1171)\end{array}$ & & \\
\hline$W$ & $\begin{array}{c}\hat{\mu}=0.0939 \\
(0.0191)\end{array}$ & $\begin{array}{c}\hat{\beta}=1.0478 \\
(0.0676)\end{array}$ & & & \\
\hline$E E_{x}$ & $\begin{array}{c}\hat{\alpha}=0.1212 \\
(0.0136)\end{array}$ & $\begin{array}{c}\hat{\lambda}=1.2180 \\
(0.1488)\end{array}$ & ------------ & ------ & \\
\hline$E_{x}$ & $\begin{array}{c}\hat{\alpha}=0.1068 \\
(0.0094)\end{array}$ & (1) & $\ldots$ & $\cdots$ & \\
\hline$E \boldsymbol{W}$ & $\begin{array}{c}\hat{\mu}=0.4538 \\
(0.2398)\end{array}$ & $\begin{array}{c}\hat{\beta}=0.6544 \\
(0.1346)\end{array}$ & $\begin{array}{c}\hat{\lambda}=2.7965 \\
(1.2633)\end{array}$ & & \\
\hline $\boldsymbol{R}$ & $\begin{array}{c}\hat{\mu}=0.0051 \\
(0.0004)\end{array}$ & $\ldots$ & & - & \\
\hline
\end{tabular}


Figure 3 displays: (a) the estimated densities of the $E A W, K w-M W, M W, W, E E, E$, $E W, L$ and $R$ distributions for the data, and $(b)$ the estimated cdf from the fitted the $E A W$, Kumaraswamy modified Weibull distribution $(K w-M W)$ (Cordeiro et al., (2014)), $M W, W, E E_{x}, E_{x}, E W$ and $R$ distributions and the empirical cdf of the data set. These results indicate that the $E A W$ model has the lowest values of $W^{*}, A^{*}$ and $K-S$ statistics and the largest $K-S p$-value, among the fitted distributions, and therefore it could be chosen as the best model.

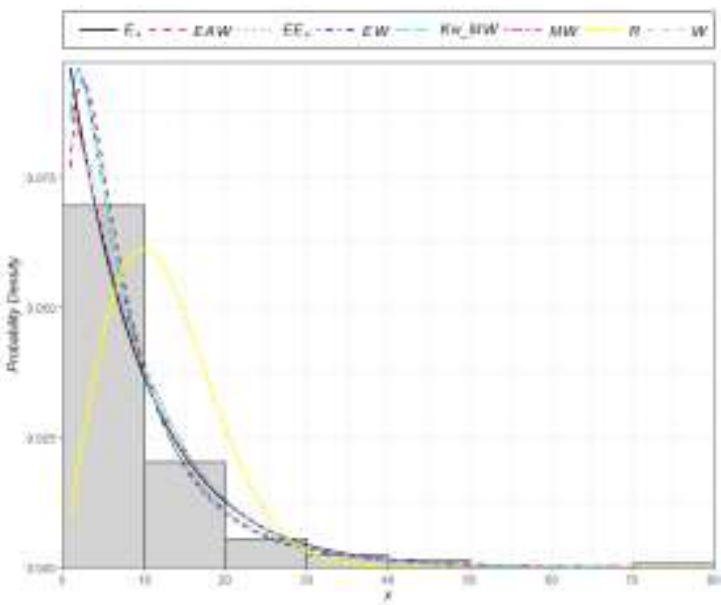

(a)

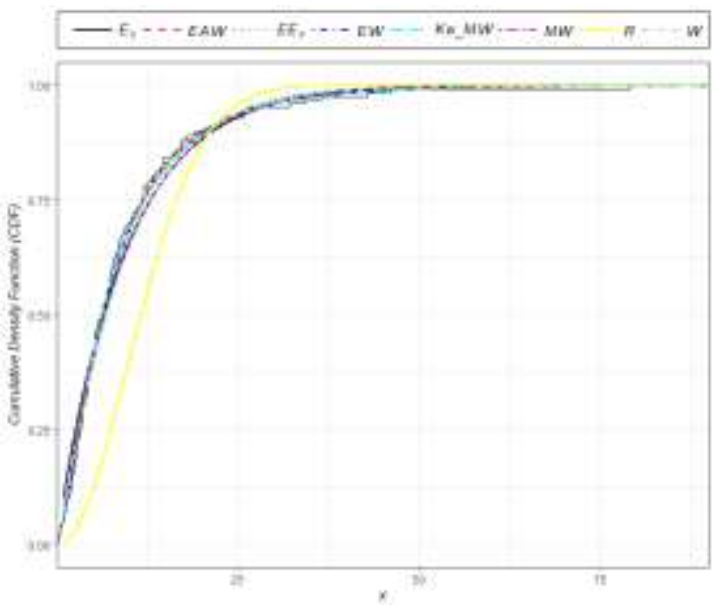

(b)

Figure 3: The estimated densities (a) and the estimated cdf $(b)$ of the $\boldsymbol{E} \boldsymbol{A} \boldsymbol{W}$ distribution and other estimated distributions, for the bladder cancer data.

\section{References}

1. Bain L.J. (1974). Analysis for the linear failure -rate life- testing distribution. Technometrics; 16 (4): 551-9.

2. Bakouch, H. S., Al-Zahrani, B. M., Al-Shomrani, A, Marchi, V. A and Louzada, F. (2011). An extended lindley distribution. Journal of the Korean Statistical Society, 41(1): 75-85.

3. Barriga, G. D. C., Louzada, F. and Cancho, V. G. (2011). The Complementary Exponential Power lifetime model. Computational Statistics and Data Analysis, 54(5): 1250-1259.

4. Bebbington MS, Lai CD, and Zitikis, R. (2007). Aflexible Weibull extension. Reliability Engineering and System Safety. 92 (6), 719-26.

5. Bonferroni C.E. (1930). Elmenti di statistica generale. Libreria Seber, Firenze.

6. Choulakian, V., Stephens, MA. (2001). Goodness-of-fit for the generalized Pareto distribution, Technometrics. 43 (4), 478-484.

7. Cordeiro G. M., Ortega E. M., and Silva G. (2014). The Kumaraswamy modified Weibull distribution: theory and applications. Journal of Statistical Computation and Simulation. 84 (7), 1387-1411. 
8. Development Core Team, R. A. (2012). Language and Environment for Statistical Computing, R Foundation for Statistical Computing, Vienna, Austria.

9. Elbatal. I (2011) Exponentiated modified Weibull distribution. Economic Quality Control. 26, 189-200.

10. Gupta, P.L. and Gupta,R.C (1983). On the moments of residual life in reliability and some characterization results, Communications in Statistics-Theory and Methods, 12, 449-461.

11. Gupta, R.D, Kundu, D.(2001). Exponentiated exponential distribution. An alternative to gamma and Weibull distributions. Biometrical Journal 43, 117-130.

12. Kundu, C., Nanda, A. K. (2010). Some reliability properties of the inactivity time. Communications in StatisticsTheory and Methods, 39, 899-911.

13. Lai, C.D, Xie, M, Murthy, D.N.P.(2003). A modified Weibull distribution. IEEE Transactions on Reliability 52, 33-37.

14. Lee, E. and Wang. J. (2003). Statistical methods for survival data analysis. 3. New York: Wiley.

15. Miller, J.R.G. (1981). Survival Analysis,Wiley,New York.

16. Mudholkar GS, Srivastava D K. (1993). Exponentiated Weibull family for analyzing bathtub failure- rate data. IEEETransactions on Reliability. 42(2): 299302.

17. Mudholkar G, Srivastava D, Kollia G. (1996) A Generalization of the Weibull Distribution with Application to the Analysis of Survival Data. Journal of the American Statistical Association. 91, 1575-1583.

18. Nadarajah, S. and Kotz, S. (2006). The exponentiated type distributions. Acta Applicandae Mathematicae, 92, 97-11.

19. Pham, H., Lai, C.D.(2007). On recent generalizations of the Weibull distribution. IEEE Transactions on Reliability 56, 454-458.

20. Sarhan, A and Kundu, D (2009) Generalized linear failure rate distribution, Commun. Statist. Theory Methods 38 (5), 642-660.

21. Sarhan A M, and Zaindin M. (2009). Modified Weibull distribution. Applied Sciences .11, $123-36$.

22. Smith, R. M. \& Bain, L. J. (1975). An exponential power life-testing distribution. Communications in Statistics - Theory and Methods, 4, 469-481.

23. Weibull W. A. (1951). Statistical distribution function of wide applicability.Journal of Applied Mechanics. 18, 293-297.

24. Xie, M. and Lai, C. D. (1995). Reliability analysis using an additive Weibull model with bathtub-shaped failure rate function. Reliability Engineering and System Safety, 52, 87-93.

25. Zhang T,Xie M. (2011). On the upper truncated Weibull distribution and its reliability implications. Reliability Engineering and System Safety, 96 (1), 194200. 


\section{Appendix}

Let $S(x)=e^{-\left(\alpha x^{\theta}+\mu x^{\beta}\right)}$, the entries of the matrix, $I_{n}(\boldsymbol{\phi})$, in (28), are

$$
\begin{aligned}
& I_{\alpha \alpha}=\frac{\partial^{2} \ell_{n}}{\partial \alpha^{2}}=\lambda(1-S(x))^{\lambda-3} S(x) x^{2 \theta-1}\left[\left(\theta\left(x^{\theta} \alpha-2\right)+\beta \mu x^{\beta}\right)+\lambda S^{2}(x)\left(\theta\left(\alpha \lambda x^{\theta}-2\right)+\beta \lambda \mu x^{\beta}\right)\right. \\
& \left.+S(x)\left(\theta\left(2(1+\lambda)+\alpha x^{\theta}(1-3 \lambda)\right)+\mu \beta(1-3 \lambda) x^{\beta}\right)\right] \text {, } \\
& I_{\theta \theta}=\frac{\partial^{2} \ell_{n}}{\partial \theta^{2}}=\alpha \lambda(1-S(x))^{\lambda-3} \log (x) S(x) x^{\theta-1}\left[2(1-S(x))\left(\left(1-\alpha x^{\theta}\right)+S(x)\left(\alpha \lambda x^{\theta}-1\right)\right)\right. \\
& +\log (x)\left(\theta \left[S^{2}(x)+\left(1+\alpha x^{\theta}\left(\alpha x^{\theta}-3\right)\right)+\alpha \lambda x^{\theta} S^{2}(x)\left(\alpha \lambda x^{\theta}-3\right)\right.\right. \\
& \left.+S(x)\left(\alpha x^{\theta}\left(3(\lambda+1)+\alpha(1-3 \lambda) x^{\theta}\right)-2\right)\right] \\
& \left.\left.+\beta \mu x^{\beta}\left[\left(\alpha x^{\theta}-1\right)+\lambda\left(\alpha \lambda x^{\theta}-1\right)+S(x)\left(1+\lambda+\alpha(1-3 \lambda) x^{\theta}\right)\right]\right)\right] \text {, } \\
& I_{\mu \mu}=\frac{\partial^{2} \ell_{n}}{\partial \mu^{2}}=\lambda(1-S(x))^{\lambda-3} S(x) x^{2 \beta-1}\left[\left(\beta\left(\mu x^{\beta}-2\right)+\alpha \theta x^{\theta}\right)+\lambda S^{2}(x)\left(\beta\left(\lambda \mu x^{\beta}-2\right)+\alpha \theta \lambda x^{\theta}\right)\right. \\
& \left.+S(x)\left(\beta\left(2(1+\lambda)+\mu x^{\beta}(1-3 \lambda)\right)+\alpha \theta(1-3 \lambda) x^{\theta}\right)\right], \\
& I_{\beta \beta}=\frac{\partial^{2} \ell_{n}}{\partial \beta^{2}}=\mu \lambda(1-S(x))^{\lambda-3} \log (x) S(x) x^{\beta-1}\left[2(1-S(x))\left(\left(1-\mu x^{\beta}\right)+S(x)\left(\mu \lambda x^{\beta}-1\right)\right)\right. \\
& +\log (x)\left(\beta \left[S^{2}(x)+\left(1+\mu x^{\beta}\left(\mu x^{\beta}-3\right)\right)+\mu \lambda x^{\beta} S^{2}(x)\left(\mu \lambda x^{\beta}-3\right)\right.\right. \\
& \left.+S(x)\left(\mu x^{\beta}\left(3(\lambda+1)+\mu(1-3 \lambda) x^{\beta}\right)-2\right)\right] \\
& \left.\left.+\alpha \theta x^{\theta}\left[\left(\mu x^{\beta}-1\right)+\lambda\left(\mu \lambda x^{\beta}-1\right)+S(x)\left(1+\lambda+\mu(1-3 \lambda) x^{\beta}\right)\right]\right)\right] \text {, } \\
& I_{\lambda \lambda}=\frac{\partial^{2} \ell_{n}}{\partial \lambda^{2}}=S(x) x^{-1}(1-S(x))^{\lambda-1}\left(\alpha \theta x^{\theta}+\beta \mu x^{\beta}\right) \log (1-S(x))(\lambda \log (1-S(x))+2), \\
& I_{\alpha \theta}=\frac{\partial^{2} \ell_{n}}{\partial \alpha \partial \theta} \\
& =\frac{\partial^{2} \ell_{n}}{\partial \theta \partial \alpha}=\lambda S(x)(1-S(x))^{\lambda-3} x^{\theta-1}\left[(1-S(x))\left(\left(1-\alpha x^{\theta}\right)+S(x)\left(\alpha \lambda x^{\theta}-1\right)\right)\right. \\
& +\log (x)\left(\theta \left[S^{2}(x)+\left(1+\alpha x^{\theta}\left(\alpha x^{\theta}-3\right)\right)+S^{2}(x) \alpha \lambda x^{\theta}\left(\alpha \lambda x^{\theta}-3\right)\right.\right. \\
& \left.+S(x)\left(\alpha x^{\theta}\left(3(1+\lambda)+\alpha x^{\theta}(1-3 \lambda)\right)-2\right)\right] \\
& \left.\left.+\beta \mu x^{\beta}\left[\left(\alpha x^{\theta}-1\right)+\lambda S^{2}(x)\left(\alpha \lambda x^{\theta}-1\right)+S(x)\left(1+\lambda+\alpha x^{\theta}(1-3 \lambda)\right)\right]\right)\right] \text {, } \\
& I_{\alpha \mu}=\frac{\partial^{2} \ell_{n}}{\partial \alpha \partial \mu} \\
& =\frac{\partial^{2} \ell_{n}}{\partial \mu \partial \alpha}=S(x)(1-S(x))^{\lambda-3} x^{\beta+\theta-1} \lambda\left[\left(\left(\alpha x^{\theta}-1\right) \theta+\beta\left(-1+x^{\beta} \mu\right)\right)\right. \\
& +S(x)\left(\theta\left(1+\lambda+x^{\theta} \alpha(1-3 \lambda)\right)+\beta\left(1+\lambda+\mu x^{\beta}(1-3 \lambda)\right)\right) \\
& \left.+S^{2}(x) \lambda\left(\theta\left(\alpha \lambda x^{\theta}-1\right)+\beta\left(\lambda \mu x^{\beta}-1\right)\right)\right] \text {, }
\end{aligned}
$$




$$
\begin{aligned}
& =\frac{\partial^{2} \ell_{n}}{\partial \beta \partial \alpha}=\lambda \mu S(x)(1-S(x))^{\lambda-3} x^{\beta+\theta-1}[(1-S(x))(\lambda S(x)-1) \\
& +\log (x)\left(\left(\theta\left(\alpha x^{\theta}-1\right)+\beta\left(\mu x^{\beta}-1\right)\right)\right. \\
& +S(x)\left(\theta\left(1+\lambda+\alpha x^{\theta}(1-3 \lambda)\right)+\beta\left(1+\lambda+\mu x^{\beta}(1-3 \lambda)\right)\right) \\
& \left.\left.+\lambda S^{2}(x)\left(\theta\left(\alpha \lambda x^{\theta}-1\right)+\beta\left(\lambda \mu x^{\beta}-1\right)\right)\right)\right] \\
& I_{\alpha \lambda}=\frac{\partial^{2} \ell_{n}}{\partial \alpha \partial \lambda} \\
& =\frac{\partial^{2} \ell_{n}}{\partial \lambda \partial \alpha}=-S(x)(1-S(x))^{\lambda-2} x^{\theta-1}\left[\theta\left(S(x)+\left(\alpha x^{\theta}-1\right)-2 \alpha \lambda x^{\theta} S(x)\right)+\beta \mu x^{\beta}(1-2 \lambda S(x))\right. \\
& \left.-\lambda \log (1-S(x))\left(\theta\left(\alpha x^{\theta}(\lambda S(x)-1)-S(x)+1\right)-\mu \beta x^{\beta}(1-\lambda S(x))\right)\right] \text {, } \\
& I_{\theta \mu}=\frac{\partial^{2} \ell_{n}}{\partial \theta \partial \mu} \\
& =\frac{\partial^{2} \ell_{n}}{\partial \mu \partial \theta}=\alpha \lambda S(x)(1-S(x))^{\lambda-3} x^{\beta+\theta-1}[(1-S(x))(\lambda S(x)-1) \\
& +\log (x)\left(\left(\theta\left(\alpha x^{\theta}-1\right)+\beta\left(\mu x^{\beta}-1\right)\right)\right. \\
& +S(x)\left(\theta\left(1+\lambda+\alpha x^{\theta}(1-3 \lambda)\right)+\beta\left(1+\lambda+\mu x^{\beta}(1-3 \lambda)\right)\right) \\
& \left.\left.+\lambda S^{2}(x)\left(\theta\left(\alpha \lambda x^{\theta}-1\right)+\beta\left(\lambda \mu x^{\beta}-1\right)\right)\right)\right] \text {, } \\
& I_{\mu \lambda}=\frac{\partial^{2} \ell_{n}}{\partial \mu \partial \lambda} \\
& =\frac{\partial^{2} \ell_{n}}{\partial \lambda \partial \mu}=-S(x)(1-S(x))^{\lambda-2} x^{\beta-1}\left[\alpha \theta x^{\theta}(1-2 \lambda S(x))+\beta\left(S(x)\left(1-2 \lambda \mu x^{\beta}\right)+\left(\mu x^{\beta}-1\right)\right)\right. \\
& \left.+\lambda \log (1-S(x))\left(x^{\theta} \alpha \theta(1-\lambda S(x))-\beta\left(S(x)\left(\lambda \mu x^{\beta}-1\right)+\left(1-\mu x^{\beta}\right)\right)\right)\right] \text {, } \\
& I_{\theta \beta}=\frac{\partial^{2} \ell_{n}}{\partial \theta \partial \beta} \\
& =\frac{\partial^{2} \ell_{n}}{\partial \beta \partial \theta}=\alpha \lambda \mu S(x)(1-S(x))^{\lambda-1} \log (x) x^{\beta+\theta-1}[2(1-S(x))(\lambda S(x)-1) \\
& +\log (x)\left(\left(\theta\left(\alpha x^{\theta}-1\right)+\beta\left(\mu x^{\beta}-1\right)\right)\right. \\
& +S(x)\left(\theta\left(1+\lambda+\alpha x^{\theta}(1-3 \lambda)\right)+\beta\left(1+\lambda+\mu x^{\beta}(1-3 \lambda)\right)\right) \\
& \left.\left.+\lambda S^{2}(x)\left(\theta\left(\alpha \lambda x^{\theta}-1\right)+\beta\left(\lambda \mu x^{\beta}-1\right)\right)\right)\right] \text {, } \\
& I_{\theta \lambda}=\frac{\partial^{2} \ell_{n}}{\partial \theta \partial \lambda}
\end{aligned}
$$




$$
\begin{aligned}
=\frac{\partial^{2} \ell_{n}}{\partial \lambda \partial \theta}=\alpha & S(x)(1-S(x))^{\lambda-2} x^{\theta-1}[1-S(x)(\theta \log (x)+1) \\
& +\log (x)\left(\left(\theta\left(1-\alpha x^{\theta}\right)-\beta \mu x^{\beta}\right)+2 \lambda S(x)\left(\alpha \theta x^{\theta}+\beta \mu x^{\beta}\right)\right) \\
& +\lambda \log (1-S(x))(1-S(x) \\
& \left.\left.+\log (x)\left(\theta\left(\left(1-\alpha x^{\theta}\right)+S(x)\left(\alpha \lambda x^{\theta}-1\right)\right)-\mu \beta x^{\beta}(1-\lambda S(x))\right)\right)\right]
\end{aligned}
$$

$$
\begin{aligned}
I_{\mu \beta}=\frac{\partial^{2} \ell_{n}}{\partial \mu \partial \beta} & \\
=\frac{\partial^{2} \ell_{n}}{\partial \beta \partial \mu}= & \lambda S(x)(1-S(x))^{\lambda-3} x^{\beta-1}\left[(1-S(x))\left(S(x)\left(\lambda \mu x^{\beta}-1\right)+\left(1-\mu x^{\beta}\right)\right)\right. \\
& +\log (x)\left(\alpha \theta x^{\theta}\left(\left(\mu x^{\beta}-1\right)+S(x)\left(1+\lambda+\mu x^{\beta}(1-3 \lambda)\right)+S^{2}(x) \lambda\left(\lambda \mu x^{\beta}-1\right)\right)\right. \\
& +\beta\left(S^{2}(x)\left(1+\lambda \mu x^{\beta}\left(\lambda \mu x^{\beta}-3\right)\right)+\left(1+x^{\beta} \mu\left(\mu x^{\beta}-3\right)\right)\right. \\
& \left.\left.\left.+S(x)\left(\mu x^{\beta}\left(3(1+\lambda)+\mu x^{\beta}(1-3 \lambda)\right)-2\right)\right)\right)\right]
\end{aligned}
$$

and

$$
\begin{aligned}
I_{\beta \lambda}=\frac{\partial^{2} \ell_{n}}{\partial \beta \partial \lambda} & \\
=\frac{\partial^{2} \ell_{n}}{\partial \lambda \partial \beta}=\mu & S(x)(1-S(x))^{\lambda-2} x^{\beta-1}[1-S(x)(\beta \log [x]+1) \\
& +\log (x)\left(\left(\beta\left(1-\mu x^{\beta}\right)-\alpha \theta x^{\theta}\right)+2 \lambda S(x)\left(\alpha \theta x^{\theta}+\beta \mu x^{\beta}\right)\right) \\
& +\lambda \log (1-S(x))(1-S(x) \\
& \left.+\log (x)\left(\beta\left(\left(1-x^{\beta} \mu\right)+S(x)\left(x^{\beta} \lambda \mu-1\right)\right)-\alpha \theta x^{\theta}(1-\lambda S(x))\right)\right) .
\end{aligned}
$$

